\title{
Bar code app aims to cut sugar intake
}

In this story (BMJ 2017;356:j26, doi:10.1136/bmj.j26) we refer to the new app from Pubic Health England that tells users about the amount of saturated fat, salt, and sugar in food and drinks as Sugar Smart. It is in fact called Be Food Smart. We apologise for this error. 